\title{
Open Vehicle Routing Problem by Ant Colony Optimization
}

\author{
Er. GurpreetSingh ${ }^{1}$ \\ Lecturer,Deptt. of CSE $^{1}$ \\ SBBSIET $^{1}$ Padhiana, Punjab, \\ India $^{1}$
}

\author{
Dr. Vijay Dhir ${ }^{2}$ \\ Associate Professor \& Head, Deptt. of CSE ${ }^{2}$ \\ SBBSIET $^{2}$ Padhiana,Punjab, \\ India $^{2}$
}

\begin{abstract}
Vehicle routing problem (VRP) is real-world combinatorial optimization problem which determine the optimal route of a vehicle. Generally, to provide the efficient vehicle serving to the customer through different services by visiting the number of cities or stops, the VRP follows the Travelling Salesman Problem (TSP), in which each of vehicle visiting a set of cities such that every city is visited by exactly one vehicle only once. This work proposes the Ant Colony Optimization (ACO)TSP algorithm to eliminate the tour loop for Open Vehicle routing Problem (OVRP). A key aspect of this algorithm is to plan the routes of buses that must pick up and deliver the school students from various bus stops on time, especially in the case of far distance covered by the vehicle in a rural area and find out the efficient and safe vehicle route.
\end{abstract}

Keywords-Ant Colony Optimization (ACO); Vehicle routing Problem(VRP); Open Vehicle routing Problem(OVRP); Travelling Salesman Problem(TSP);Swarm Intelligence(SI)

\section{INTRODUCTION}

The School Bus Routing Problem (SBRP) is a common real-life problem, proposed in the literature by Newton and Thomas (1969), but has not been tackled that often in the field of computer science. The problem is closely related to the Vehicle Routing Problem (VRP), which has been a popular research area for the last four decades. Vehicle Routing Problem was first described by Dantzig and Ramser (1959), and has been proved NP-hard by Lenstra and Kan (1981). Vehicle Routing Problem (VRP) is a problem which searches the optimal routes that a vehicle travels in order to serve customers residing in a geographically dispersed area. The SBRP has the same characteristics with the Vehicle Routing Problem (VRP) in several ways. While a VRP deals with freight transportation, the SBRP is related to student transportation [1]. Even that now a day many of the organisations are faced with the problem regarding efficient vehicle fleets. When, they need to pick up and deliver items and goods or to provide the pick-up and delivery services to the people. Mainly, in the case of a school transportation system, to service several students with a fleet of vehicles, at morning time pick up from their home location and deliver at central depot school and at evening time pick up from central depot school and deliver to the different home locations of the students on time.

The proposed algorithm consists of two parts; the first part introduces the ACO algorithm with tour loop elimination. The second part describes the comparison study of OVRP and
VRP to find out the most efficient route of buses and to select the bus stops of the vehicle to provide the pick-up and delivery services to the students on time. The study discussed in the paper involves the bus route of Sant Baba Bhag Singh International School situated in rural area in Padhiana, Punjab, India, where more than 1000 students are bussed to the school from different villages and cover up a far distance by the buses, which is the main problem that some buses don't return to the school.

\section{OPEN Vehicle Routing Problem}

The Open vehicle routing problem (OVRP) has received in the literature relatively less attention than the VRP. The problem is first described in a paper by Schrage (1981) without any solution attempt. Bodin et al. (1983) address the express airmail distribution in the USA and solve two separate routing problems, one for the delivery and another one for the pick up using a modified Clarke-Wright saving algorithm. The open vehicle routing problem (OVRP) describes efficient routes with minimum total distance and cost for a fleet of vehicles that serve some commodity to a given number of customers. E

ach customer is visited exactly once by one vehicle, while vehicle activity is bounded by capacity constraints, duration constraints and time constraints. Either each route is a sequence of customers who starts at the depot and finishes at one of the customers to whom the goods are delivered, or each route is a sequence of customers who begins at a certain customer and ends at the distribution depot, where goods are gathered. The OVRP differs from the well-known vehicle routing problem (VRP) in that the vehicles do not necessarily return to their original locations after serving to the customers; if they do, they must follow the same path in the reverse order.

The major difference in theory between the OVRP and the VRP is that the routes in the OVRP consist of Hamiltonian paths originating at the depot and ending at one of the customer side, while the routes in the VRP are Hamiltonian cycles. In other words, the best Hamiltonian path is NP-hard, since the Hamiltonian path problem is equivalent to the traveling salesman problem, which is known to be NP-hard.

The best Hamiltonian path problem with a fixed source node must be solved for each vehicle in the OVRP, and OVRP solutions involve finding the best Hamiltonian path for each set of customers assigned to a vehicle. Consequently, the OVRP is also an NP-hard problem. 


\section{LITERATURE REVIEW}

As mentioned in the introductory section of the paper, the OVRP is an NP-hard combinatorial optimization problem as to deal with OVRP instances of real time and have received in the literature relatively less attention than the VRP. There are several researchers who propose different methods for OVRP and VRP to reduce the distance and cost of the transportation. Marco Dorigo and Luca Maria Gambardella 1997 [2]"Ant Colony System: A Cooperative Learning Approach to the Traveling Salesman Problem, "introduces ant colony system (ACS)and presents an intuitive explanation of how ACS works, a distributed algorithm that is applied to the traveling salesman problem (TSP). In ACS, a set of cooperating agents called ants cooperate to find good solutions to TSP.

Ants cooperate using an indirect form of communication mediated by pheromone, deposit on the edges of the TSP graph while building solutions. Patrick Schittekat, Marc Sevaux and Kenneth Sirensen 2006 [3]" A mathematical formulation for a school bus routing problem," introduce the school bus routing problem in this paper, is similar to the standard vehicle routing problem. In the standard VRP all stops to visit are given. In our school bus routing problem, we assume that set of potential stops is given, as well as a set of students who can walk to more of these potential stops. The school buses used to pick up the students and transport them to their schools have a finite capacity. The goal of this routing problem is to select a subset of stops that will actually be visited by the buses, determine which stop each student should walk to and develop a set of tours that minimize the total distance travelled by all buses. Jean-Yves Potvin, Ying X, Ilham Benyahia 2006 [4] presents a dynamic vehicle routing and scheduling problem with time windows is described where both real-time customer requests and dynamic travel times are considered The D Aksen, Z Özyurt1 and N Aras 2007 [5], consider a variant of the open vehicle routing problem in which vehicles depart from the depot, visit a set of customers and end their routes at special nodes called driver nodes. A driver node can be the home of the driver or a parking lot where the vehicle will stay overnight. AN Letch ford, JLysgaard and RW Eglese 2007[6] also classifies the open vehicle routing problems, the vehicles are not required to return to the depot after completing service and they are use the integer programming method based on branch and cut.

Ashek Ahmmed, Md. Ali Ahsan Rana, Abul Ahsan Md. Mahmudul Haque, Md. Al Mamun 2008 [7] presents A Multiple Ant Colony Optimization based approach useful to solve dynamic vehicle routing problems with time windows. MACS-DVRPTW is organized with a hierarchy of artificial ant colonies designed to successively optimize a multiple objective function. A.E. Rizzoli, R. Montemanni ,E. Lucibello ,L.M. Gambardella 2007 [8] presents the applications of ACO to a number of real-world problems: a VRP with time windows for a major supermarket chain in Switzerland; a VRP with pickup and delivery for a leading distribution company in Italy; a time dependent VRP for freight distribution in the city of Padua, Italy, where the travel times depend on the time of the day; and an on-line VRP in the city of Lugano, Switzerland, where customers' orders arrive during the delivery process. Wen-Chen $\mathrm{Hu}$, Naima Kaabouch, Lei Chen, and Hung-Jen Yang 2011 [9] "Incremental Location Searching for Route Anomaly Detection," presents location-based research, which uses location information to find route anomalies, a common problem of daily life. For example, an alert should be generated when a school bus misses part of a route. Different kinds of route anomalies are discussed and various methods for detecting the anomalies are proposed. The major methods use a technique of incremental location search, which finds matched routes as the search route is entered location by location. An alert is generated when no matched routes exist.

Juan S. Arias-Rojas, José Fernando Jiménez,Jairo R. Montoya-Torres 2012 [10]'Solving of School Bus Routing Problem By Ant Colony Optimization, "presents The school bus routing problem (SBRP) seeks to plan an efficient schedule of a fleet of school buses that must pick up students from various bus stops and deliver them by satisfying various constraints. Jianyong Jin, Teodor Gabriel Crainic and Arne Løkketangen 2012 [11]“A parallel multi-neighbourhood cooperative tabu search for capacitated vehicle routing problems" presents a parallel tabu search algorithm that utilizes several different neighbourhood structures for solving the capacitated vehicle routing problem. Single neighbourhood or neighbourhood combinations are encapsulated in tabu search threads and they cooperate through a solution pool for the purpose of exploiting their joint power.P. Schittekat, J. Kinable, K. Sörensen, M. Sevaux, F. Spieksma, J. Springael 2013 [12] presents the paper on understanding the joint problem of bus route generation and bus stop selection, which determine the set of stops to visit, determine for each student which stop, determine for each student which Stop. Taehyeong Kim and Bum-Jin Park 2013 [13]" Model and Algorithm for Solving School Bus Problem" presents the model for school bus routing problem is proposed, and a heuristic algorithm for solving the proposed model is suggested. The model is formulated as a mixed-integer programming problem. School bus routing problem has been a significant concern of most people related to school and school bus system as one of the vehicle routing problems. Making an appropriate problem formulation depend on how to reflect the realities of the problem. And, as the problem scope becomes wider, the problem can't be solved only with the exact methods. So, there is a need to develop an efficient heuristic method to solve the more complicated problem. John Awuah Addor, Samuel Kwame Amponsah, Jonathan Annan and Charles Sebil 2013 [14] " School Bus Routing: A Case Study of Wood Bridge School Complex, Sekondi-Takoradi, Ghana," presents a school bus routing problem of wood bridge school complex Sekondi-Takoradi, Ghana. The problem was formulated as an integer programming model and an ant colony based metaheuristic for the travelling salesman problem was used to solve the problem.

\section{Aco-Travelling Salesman Problem}

In the early 1990s, ant colony optimization (ACO) was introduced by $\mathrm{M}$. Dorigo and colleagues as a novel natureinspired meta-heuristic for the solution of hard combinatorial optimization (CO) problems. ACO belongs to the class of meta-heuristics, which are approximate algorithms used to obtain good enough solutions to hard Combinatorial 
Optimization problems in a reasonable amount of computation time [15]. The inspiring source of ACO is the foraging behaviour of real ants. The first ACO algorithm was called the Ant system [16], and it was aimed to solve the travelling salesman problem, in which the goal is to find the shortest round-trip to link a series of cities. These are the popular ways to illustrate the ACO meta-heuristic working through the application of the traveling salesman problem (TSP) [17]. Travelling salesman problem (TSP) consists of finding the shortest route in complete weighted graph $\mathrm{G}$ with $\mathrm{n}$ nodes and $\mathrm{n}(\mathrm{n}-1)$ edges; so that the start node and the end node are identical and all other nodes in this tour are visited exactly once. The TSP consists of set of cities is given and the distance among each of them is known.

The goal is to find the shortest tour that allows each city to be visited once and only once. More generally, the goal is to find Hamiltonian tour of minimal length on a fully connected graph (Hamiltonian circuit) [18].In ant colony optimization, the problem is tackled by simulating a number of artificial ants moving on a graph that encodes the problem itself: each vertex represents a city, and each edge represents a connection between two cities. A variable called pheromone is associated with each edge and can be read and modified by ants. The most popular practical application of TSP is a regular distribution of goods or resources, finding of the shortest of customer servicing route, planning bus lines, etc., but also in the areas that have nothing to do with travel routes [19].The Moving of ant depends on the amount of pheromone updating on the graph edges and the transition probability $\mathrm{p}_{\mathrm{ij}}{ }^{\mathrm{k}}(\mathrm{t})$ of a virtual ant from the node $\mathrm{i}$ to $\mathrm{j}$ is given by the formula [16]:

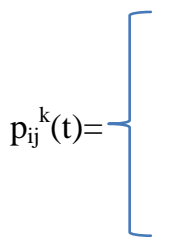

$$
\begin{aligned}
& {\left[\mathrm{T}_{\mathrm{ij}}(\mathrm{t})\right]^{\alpha} \cdot\left[\prod_{\mathrm{ij}}\right]^{\beta}}
\end{aligned}
$$

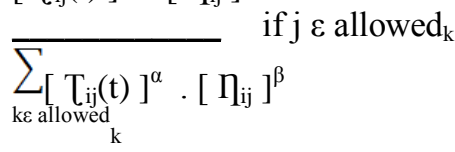

$$
\begin{aligned}
& 0 \text { otherwise }
\end{aligned}
$$

Where, $T_{\mathrm{ij}}$ represents the pheromone trail, $\eta_{\mathrm{ij}}$ is the inverse distance, the parameters $\alpha$ and $\beta$ control the balance to which ants follow the closest cities and the strongest pheromone trails.

\section{A. Proposed ant colony algorithm (aco) for ovrp}

This algorithm proposes the ACO tour loop elimination, which is further used to describe the Open vehicle routing problem. Loop elimination can be done by iteratively scanning the node identifiers position by position from node ( $i$ to $j$ ) and node ( $\mathrm{j}$ to $\mathrm{i}$ ) and pictorial dataset are used in the form of map images to mark up the nodes or stops of vehicles. We can plan the vehicle fleet through using an ant colony optimization algorithm by formulating the problem in the form of TSP model for OVRP.

Given a set of $n$ cities (stops), ACO-TSP for OVRP can be stated as the problem of finding a minimal lengthy open tour that visits each city once. An instance of TSP can be represented by a graph $\mathrm{G}=(\mathrm{V}, \mathrm{E})$ where $\mathrm{V}$ is the set of nodes, and $E$ is the set of arcs in the graph. Each node represents a stop in which students (customers in the classical VRP) have to be serviced, while an arc corresponds to the route to go from node $i$ to $j$. We call $\mathrm{d}_{\mathrm{ij}}$ the length of a path between cityi and $\mathrm{j}, \mathrm{d}_{\mathrm{ij}}$ can be defined in the Euclidean space and is given as follows:

$$
\mathrm{d}_{\mathrm{ij}}=\left(\left[\left(\mathrm{x}_{\mathrm{i}-\mathrm{x}} \mathrm{x}\right)^{2}+\left(\mathrm{y}_{\mathrm{i}}-\mathrm{y}_{\mathrm{j}}\right)^{2}\right]^{1 / 2}\right)
$$

Let $\mathrm{a}_{\mathrm{i}}(\mathrm{t})(\mathrm{i}=$ starting point + intermediate point + end point $)$ be the number of ants in city $\mathrm{i}$ at time $\mathrm{t}$ and let $\mathrm{m}=\sum_{i=1}^{n} S_{\mathrm{i}}(\mathrm{t})+\sum_{i=1}^{n} E_{\mathrm{i}}(\mathrm{t}) \sum_{i=1}^{n} I_{\mathrm{i}}(\mathrm{t})$ be the total number of ants. Where to start point $\left(\mathrm{S}_{\mathrm{i}}\right)$ represents source city and end point $\left(\mathrm{E}_{\mathrm{i}}\right)$ represents destination city, which are identical (value is always 1). Intermediate Point $\left(\mathrm{I}_{\mathrm{i}}\right)$ represents the cities between $\mathrm{S}_{\mathrm{i}}$ and $\mathrm{E}_{\mathrm{i} \text { i.e. }}$ (Total cities- $\mathrm{S}_{\mathrm{i}}-\mathrm{E}_{\mathrm{i}}$ ).

\section{B. ACO based solution to eliminate tour loop}

1) Initialize: To mark up the cities according to ai $(t)(i)$.

2) Set the parameters: $t=0$ \{t is the time counter $\}$ and $N C=0\{N C$ is the cycle counter of iterations)

3) To set the Trail value $\Delta T_{i j}=c$ for trail intensity and $\Delta T_{i j}=0$.

4) Set $z=1\{z$ is the tabu list index $\}$

For $\mathrm{k}=1$ to $\mathrm{m}$ do

Store the source city of $\mathrm{k}$-th ant in $\operatorname{tabu}_{\mathrm{k}}(\mathrm{z})$

5) Repeat until tabu list is full and repeated upto(n-1)

times

Set $\mathrm{z}=\mathrm{z}+1$

For $\mathrm{k}=1$ to $\mathrm{m}$ do

Choose the next city $\mathrm{j}$ to move with probability according to equation (1

Then move the the $\mathrm{k}$-th ant to the next city $\mathrm{j}$ and update city $\mathrm{j}$ in tabu list

6) For $k=1$ to $m$ do

Move the k-th ant from $\operatorname{tabu}_{\mathrm{k}}(\mathrm{n})$ to tabu(1)

7) Initialize the tour:

For ic $=1$ to $n$-ants, where ic is number of cities

Tour (i) =tour (1 to $\mathrm{n}-2)$

Update tabu(n-1) to tabu(2)

Tour $=$ Tour +2

Tour (n-ants) $=2$

Tour $(2, n-1)=$ Tour $(1$ to $n-2)$

Tour $(1)=1$

Update the shortest tour found

8) Pheromone update on every edge $(i, j)$ for $k=1$ to $m$, according to the following equation:

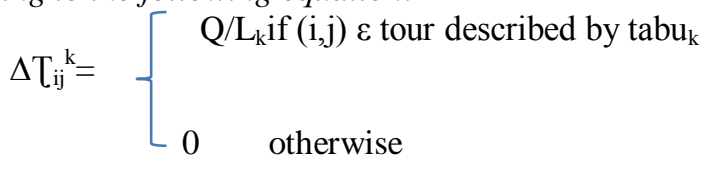

$\Delta \mathrm{T}_{\mathrm{ij}}=\Delta \mathrm{T}_{\mathrm{ij}}+\Delta \mathrm{T}_{\mathrm{ij}}^{\mathrm{k}}$

9) For every edge $(i, j)$ compute $T_{i j}(t+n)$ according to equation: $T_{i j}(t+n)=\rho . T_{i j}(t)+\Delta T_{i j}$

Set $\mathrm{t}=\mathrm{t}+\mathrm{n}$

Set $\mathrm{NC}=\mathrm{NC}+1$

For every edge $(\mathrm{i}, \mathrm{j})$ set $\Delta \mathrm{T}_{\mathrm{ij}=0}$

10) If NC greater than assign value 
then

Empty the all tabu lists GOTO Step 4

else

Print shortest tour

Stop

\section{Simulation Results}

The algorithm is coded in MATLAB-2011b to achieve the computational optimize results. The results are described in two cases: Case-I describe the ACO tour loop elimination and in Case-II, analyses the path selection and stops/nodes selection behaviour of the two different ACO algorithms-one with tour cycle and others with tour loop elimination. To describe the results of the proposed algorithm or to show up the ACO tour loop elimination, the algorithm is tested by using the dataset in the form of as map images. In map images, the user has to select the stops by pointing them wherever required. The three types of points are given-red, green and black. Where, red point describes the starting/source position of the bus; green point describes the different stops of bus, and black point describes the end/destination position of the bus.

In the $\mathrm{ACO}$, ant colony system with tour loop elimination is used to evaluate the result, a value for each parameter is: $\alpha=2, \beta=6, \rho=0.5, Q=2.7179$ and select the number of ants equal to the number of bus stops(including red and black point).

\section{A. Case-I:}

The result of algorithm ACO-tour loop elimination as shown in Fig.1, where a number of stops and number of ants are 21 and iterations $=1000$ and the final open route is:

$1 \rightarrow 3 \rightarrow 4 \rightarrow 5 \rightarrow 6 \rightarrow 7 \rightarrow 8 \rightarrow 9 \rightarrow 10 \rightarrow 11 \rightarrow 12 \rightarrow 13 \rightarrow 14 \rightarrow 15 \rightarrow 16$ $\rightarrow 17 \rightarrow 18 \rightarrow 19 \rightarrow 20 \rightarrow 21 \rightarrow 2$

Point 1 (red) represents the starting/source position, and point 2 (black) represents the end/destination position of the vehicle.

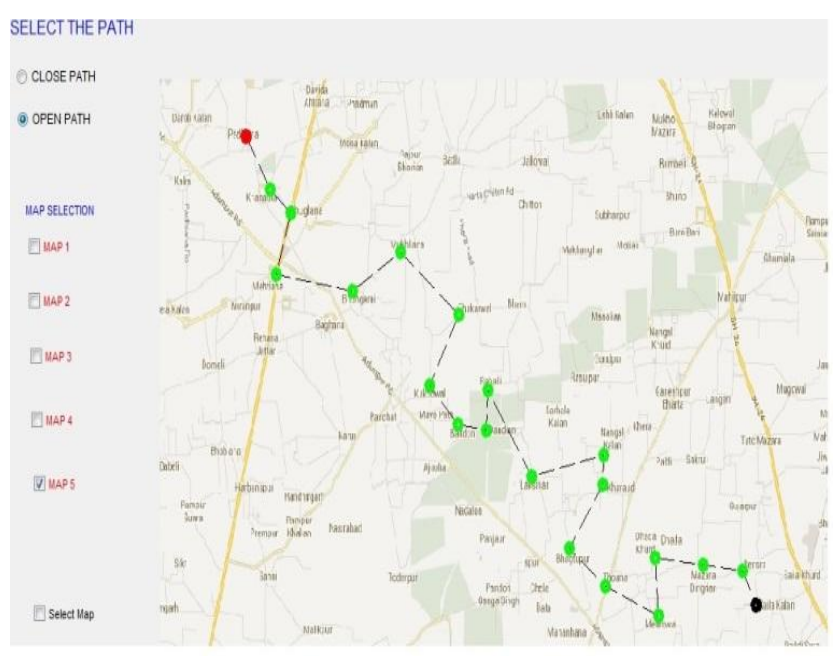

Fig. 1. shows the route map of ACO tour loop elimination

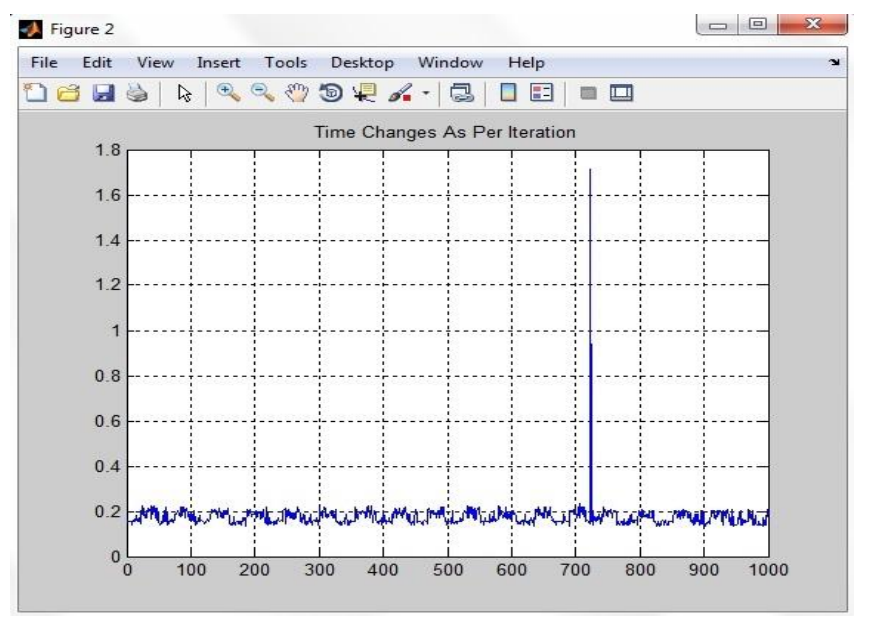

Fig. 2. shows the time changes as per iteration

\section{B. Case-II:}

The computational results of the ACO algorithm-one with tour cycle and others with tour loop elimination obtained are shown in Table 1.

TABLE I. ACO RESULT

\begin{tabular}{|c|c|c|}
\hline & \multicolumn{2}{|c|}{ ACO Result } \\
\hline & $\begin{array}{l}\text { Existing ACO } \\
\text { Algorithm }\end{array}$ & $\begin{array}{l}\text { Proposed } \\
\text { ACO } \\
\quad \text { Algorithm } \\
\text { (Tour Loop } \\
\text { Elimination) }\end{array}$ \\
\hline $\begin{array}{l}\text { Computational } \\
\text { Distance (miles) }\end{array}$ & 1833.2729 & 1329.8818 \\
\hline $\begin{array}{c}\text { Computational } \\
\text { Time (millisecond) }\end{array}$ & 0.201 & 0.147 \\
\hline No. of Iterations & 5000 & 1000 \\
\hline Tour length & $\begin{array}{c}18 \rightarrow 17 \rightarrow 16 \rightarrow 1 \\
5 \rightarrow 14 \rightarrow \\
\\
19 \rightarrow 20 \rightarrow 10 \rightarrow 1 \\
1 \rightarrow 12 \rightarrow \\
13 \rightarrow 7 \rightarrow 9 \rightarrow 8 \rightarrow \\
21 \rightarrow 4 \rightarrow \\
3 \rightarrow 2 \rightarrow 1 \rightarrow 6 \rightarrow 5 \\
\rightarrow 18\end{array}$ & $\begin{aligned} & 1 \rightarrow 3 \rightarrow 4 \rightarrow 5 \rightarrow 6 \rightarrow \\
& 7 \rightarrow \\
& 8 \rightarrow 9 \rightarrow 10 \rightarrow 11 \rightarrow 1 \\
& 2 \rightarrow \\
& 13 \rightarrow 14 \rightarrow 15 \rightarrow 16 \\
& \rightarrow 17 \rightarrow \\
& 18 \rightarrow 19 \rightarrow 20 \rightarrow 21 \\
& \rightarrow 2\end{aligned}$ \\
\hline No. of ants & 21 & 21 \\
\hline Q & 2.7179 & 2.7179 \\
\hline$\alpha$ & 2 & 2 \\
\hline$\beta$ & 6 & 6 \\
\hline$\rho$ & 0.5 & 0.5 \\
\hline
\end{tabular}


We can reach a summing-up to select the bus route, which one is the best either close path or open path by comparing the data in Table 1 and Fig. 3 and fig. 5, which shows the optimal solution for path selection to provide the pick-up and delivery services to the students. In fig. 5, it is depicted that some destinations are nearerto the school and some are far. However, the students belonging to an area which is away from school, reach their home earlier than the near ones.Fig. 3 gives solution to the problem.Fig. 3 also depicts that there is one feature, if end/destination position selected by the user is not accurate,then it 1 ll finalize by the method. All of these show that this method can be a better way to find out the efficient vehicle route in case of far distance covered by the vehicle.

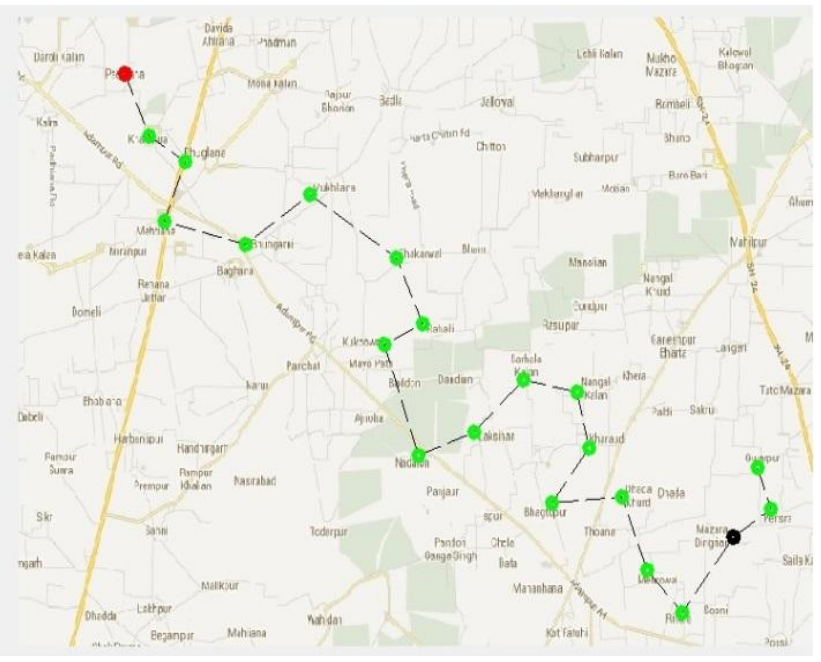

Fig. 3. Shows the open tour length

$1 \rightarrow 3 \rightarrow 4 \rightarrow 5 \rightarrow 6 \rightarrow 7 \rightarrow 8 \rightarrow 9 \rightarrow 10 \rightarrow 11 \rightarrow 12 \rightarrow 13 \rightarrow 14 \rightarrow 15 \rightarrow 16$ $\rightarrow 17 \rightarrow 18 \rightarrow 19 \rightarrow 20 \rightarrow 21 \rightarrow 2$

Point 1 (red) represents the starting/source position and point 2 (black) represents the end/destination position of the vehicle.

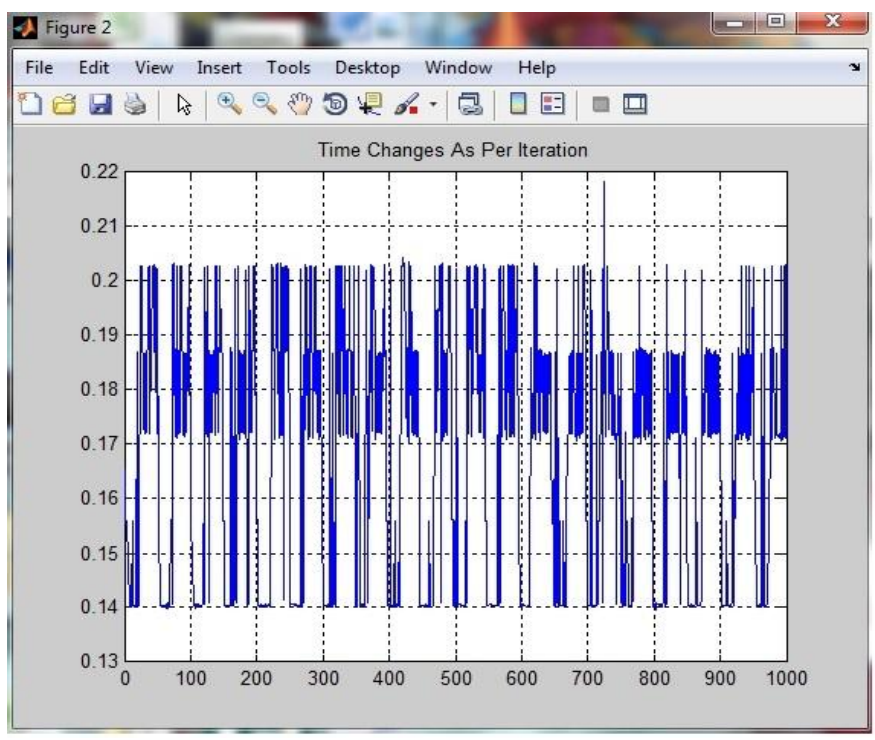

Fig. 4. shows the time changes as per iteration

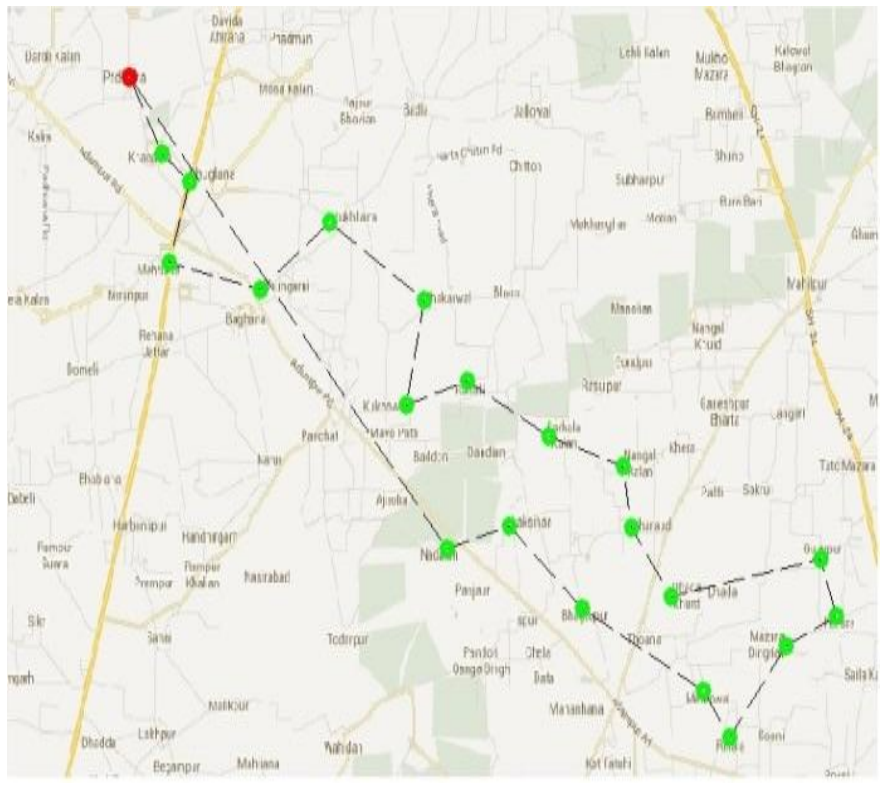

Fig. 5. shows the close tour length

$18 \rightarrow 17 \rightarrow 16 \rightarrow 15 \rightarrow 14 \rightarrow 19 \rightarrow 20 \rightarrow 10 \rightarrow 11 \rightarrow 12 \rightarrow 13 \rightarrow 7 \rightarrow 9 \rightarrow 8$ $\rightarrow 21 \rightarrow 4 \rightarrow 3 \rightarrow 2 \rightarrow 1 \rightarrow 6 \rightarrow 5 \rightarrow 18$

Point 18 (red) represents the source and destination position of vehicle.

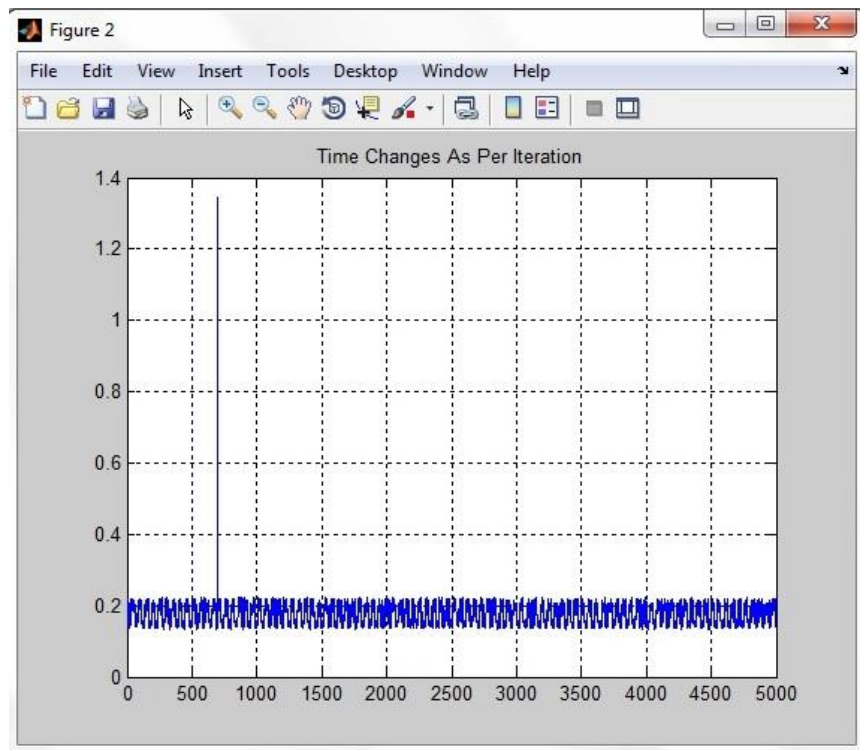

Fig. 6. shows the time changes as per iteration

\section{CONClusion AND Future SCOPE}

This paper presents an approach for solving open vehicle routing problem for school buses based on an ant colony algorithm. The proposed method is not only used to plan the close and open routes of school buses and transporter decide that which route is best to pick up and deliver the students to their home but also reduce the total distance and running of the vehicle, which further effect the transportation and operational cost of the vehicle. Computed result shows that for 
far distance; open route is best as compared to close route. Further research is to add additional feature in the proposed method to implement the ACO algorithm with navigation system, to achieve the exact route with exact timing. So, this method can be practically applied in real life.

\section{REFERENCES}

[1] Kristof Van Moffaert, Bert Van Vreckem, MihailMihaylov and Ann Now'e" A Learning Approach to the School Bus Routing Problem", Proceedings of the 23rd Benelux Conference on Artificial Intelligence, pp. $280-288$.

[2] Marco Dorigo and Luca Maria Gambardella "Ant Colony System: A Cooperative Learning Approach to the Traveling Salesman Problem", publication in the IEEE Transactions on Evolutionary Computation, Vol.1, No.1, 1997.

[3] Patrick Schittekat, Marc Sevaux, Kenneth Sirensen " A mathematical formulation for a school bus routing problem" 'I -4244-0451-7/06/2006, IEEE.

[4] Jean-Yves Potvin, Ying Xu, Ilham Benyahia "Vehicle routing and scheduling with dynamic travel times", Computers \& Operations Research 33 (2006) 1129-1137, Science Direct, Elsevier.

[5] D Aksen1, Z Özyurt and N Aras "The open vehicle routing problem with driver nodes and time deadlines" Correspondence: Necati Aras, Boğaziçi University, Department of Industrial Engineering, Bebek, 34342 İstanbul, Turkey.

[6] AN.Letchford, J Lysgaard and RW Eglese "A branch-and-cut algorithm for the capacitated open vehicle routing problem", Journal of the Operational Research Society (2007) 58, $1642-1651$.

[7] AshekAhmmed, Md. Ali Ahsan Rana, Abul Ahsan Md. MahmudulHaque, Md. Al Mamun "A Multiple Ant Colony System for Dynamic Vehicle Routing Problem with Time Window", Third 2008 International Conference on Convergence and Hybrid Information Technology.

[8] A.E. Rizzoli, R. Montemanni, E. Lucibello , L.M. Gambardella "Ant colony optimization for real-world vehicle routing problems-From theory to applications", Swarm Intell (2007) 1: 135-151 and Published online: 13 September 2007 @ Springer Science + Business Media, LLC 2007.

[9] Wen-Chen Hu, Naima Kaabouch, Lei Chen, and Hung-Jen Yang 2011" Incremental Location Searching for Route Anomaly Detection", published in 2011 IEEE International Conference on Electro/Information Technology.

[10] Juan S. Arias-Rojas,José Fernando Jiménez,Jairo R. Montoya-Torres "SOLVING OF SCHOOL BUS ROUTING PROBLEM BY ANT COLONY OPTIMIZATION" Revista EIA, ISSN 1794-1237 Número 17, p. 193-208. July 2012, Escuela de Ingeniería de Antioquia, Medellín (Colombia).

[11] JianyongJin, Teodor Gabriel Crainic and Arne Løkketangen "A parallel multi-neighborhood cooperative tabu search for capacitated vehicle routing problems", European Journal of Operational Research 222 (2012) 441-451.

[12] Schittekat, P., Kinable, J., Sörensen, K., Sevaux, M., Spieksma, F., Springael, J., A metaheuristic for the school bus routing problem with bus stop selection, European Journal of Operational Research (2013).

[13] Taehyeong Kim and Bum-Jin Park 2013" Model and Algorithm for Solving School Bus Problem", published in Journal of Emerging Trends in Computing and Information Sciences, Vol. 4, No. 8 August 2013, ISSN 2079-8407.

[14] John AwuahAddor, Samuel Kwame Amponsah, Jonathan Annan and Charles Sebil 2013 "School Bus Routing: A Case Study of Wood Bridge School Complex, Sekondi-Takoradi, Ghana",published in International Journal of Business and Social Research (IJBSR), Volume -3, No.-12, December, 2013

[15] Marco Dorigo, Christian Blum "Ant colony optimization theory: A survey", Theoretical Computer Science 344 (2005) 243 - 278,Science Direct and Elsevier.

[16] Marco Dorigo,VittorioManiezzo and Alberto Colorni "The Ant System:Optimization by a colony of cooperating agents", IEEE Transactions on Systems, Man, and Cybernetics-Part B, Vol.26, No.1, 1996, pp.1-13.

[17] Marco Dorigo and Krzysztof Socha "An Introduction to Ant Colony Optimization", IRIDIA-Technical Report Series Technical Report No.TR/IRIDIA/2006-010, ISSN 1781-3794.

[18] Marco Dorigo, Mauro Birattari, and Thomas St"utzle "Ant Colony Optimization-Artifical Ants as a computational Intelligence Technique", IEEE COMPUTATIONAL INTELLIGENCE MAGAZINE, NOVEMBER 2006.

[19] Ivan Brezina Jr, ZuzanaČičková "Solving the Travelling Salesman Problem Using the Ant Colony Optimization", Management Information Systems, Vol. 6, 4/2011, pp. 010-014. 